\title{
Os efeitos do treino de equilíbrio em crianças com paralisia cerebral diparética espástica
}

The effects of balance training in children with spastic diparetic cerebral palsy

\author{
Kátia Maria Gonçalves Allegretti ${ }^{1}$, Mirna Sayuri Kanashiro², Vanessa Costa \\ Monteiro $^{1}$, Heloise Casangi Borges ${ }^{3}$, Sissy Veloso Fontes ${ }^{4}$
}

\section{RESUMO}

Objetivo. Verificar os efeitos do treino de equilíbrio, como conduta fisioterapêutica, no ajuste postural e nas atividades funcionais de crianças com PC diparética espástica, utilizando o teste de equilíbrio de Berg e da Gross Motor Function Measure (GMFM). Método. Participaram do estudo 4 crianças, 1 menina e 3 meninos, com idade entre 7 e 8 anos. Foi utilizado um programa de treino de equilíbrio, contendo 7 atividades em diferentes superfícies (estáveis e instáveis) e a utilização de diferentes demandas sensoriais (olhos abertos e fechados). O tratamento foi realizado por um período de 3 meses consecutivos, 1 vez por semana, em sessões com duração de 60 minutos cada. Resultados. Os resultados sugerem que há possibilidade de melhora do equilíbrio funcional em crianças com PG diparética espástica com idade entre 7 e 8 anos, devido à variabilidade da prática, utilizando nas terapias diferentes atividades funcionais e promovendo a estimulação do sistema sensorial multimodal em diversos ambientes. Todas as crianças mostraram melhora no ajuste postural funcional pelo teste de equilíbrio de Berg e pelo GMFM.

Unitermos: Paralisia Cerebral. Diplegia Espástica. Equilíbrio.

Citação: Allegretti KMG, Kanashiro MS, Monteiro VC, Borges HC, Fontes SV. Os efeitos do treino de equilíbrio em crianças com paralisia cerebral diparética espástica.

\section{SUMMARY}

Objective. The objective of this study was to verify the effects of balance training and functional orthostatic activities in children with spastic diparetic cerebral palsy, using the balance tests of Berg and GMFM. Method. Four children participated in this study, 1 girl and 3 boys, aging 7 to 8 years. A protocol for balance training consisting of 7 activities based on training on different types of surfaces (stable and unstable) and using different sensorial demands (eyes open and eyes closed). The treatment was performed during a period of 3 months, once a week, with sessions lasting 60 minutes each. Results. The results suggest that improvements in functional balance in children aging 7 and 8 with cerebral palsy can occur because of the various forms of applying the treatment, using functional activities and promoting stimulation of the multimodal sensorial system in a number of environments during the different therapies. All the children showed improvements in the adjustment of functional balance after performing the Berg balance test and the GMFM.

\section{Keywords: Cerebral Palsy. Balance.}

Citation: Allegretti KMG, Kanashiro MS, Monteiro VC, Borges HC, Fontes SV. The effects of balance training in children with spastic diparetic cerebral palsy.
Trabalho realizado no Gentro de Reabilitação Lar Escola São Francisco (LESF)

1. Fisioterapeuta do LESF e Preceptora do Módulo de Neuro-infantil do Curso de Especialização em Fisioterapia Hospitalar e Ambulatorial aplicada à Neurologia - UNIFESP. Especialista em Fisioterapia Motora Hospitalar e Ambulatorial aplicada à Neurologia - UNIFESP.

2. Fisioterapeuta do LESF. Especialista em Fisioterapia Motora Hospitalar e Ambulatorial aplicada à Neurologia - UNIFESP.

3. Fisioterapeuta do LESF e Preceptora do Módulo de Neuro-adulto do Curso de Especialização em Fisioterapia Hospitalar e Ambulatorial aplicada à Neurologia - UNIFESP. Especialista em Fisioterapia Motora Hospitalar e Ambulatorial aplicada à Neurologia - UNIFESP.

4. Fisioterapeuta, Doutora em Ciências da Saúde pela UNIFESP.
Endereço para correspondência: Kátia Maria Gonçalves Allegretti Rua José Bonifácio de Andrade e Silva,150/41 São Roque-SP, CEP: 18130-003 e-mail: katiaallegretti@hotmail.com

Recebido em: 08/08/06 Revisão: 09/08/06 a 27/03/07

Aceito em: 28/03/07

Conflito de interesses: não 


\section{INTRODUÇÃO}

A paralisia cerebral $(\mathrm{PC})$ ou encefalopatia crônica não progressiva é um grupo não progressivo, mas freqüentemente mutável de distúrbio motor (tônus e postura), secundário a lesão do cérebro em desenvolvimento. $\mathrm{O}$ evento lesivo pode ocorrer no período pré, peri ou pós-natal ${ }^{1}$.

A incidência da PG no Brasil é de 2 a 3 por 1000 nascidos vivos, e a forma espástica é de maior prevalência ${ }^{2}$. Em média surgem 17.000 novos casos de PG ao ano e isso se deve também aos avanços tecnológicos e cuidados neonatais a partir dos anos 80, acarretando em aumento da sobrevivência de prematuros de muito baixo peso ao nascer, com aumento da prevalência de $\mathrm{PC}^{3,4}$.

Uma imensa variedade de problemas pode contribuir para a falta de controle postural do paciente com PC diparética espástica. O comprometimento do controle motor, incluindo alterações dos mecanismos antecipatórios (feedforward), de retroalimentação (feedback) e, algumas disfunções músculoesqueléticas afetam as reações de equilíbrio da criança diparética espástica ${ }^{5,6}$.

O treino de equilíbrio, baseado na desestabilização do paciente para que este recorra ao ajuste postural permite a utilização de estratégias de movimento postural, que são usadas, como feedback e feedforward, a fim de manter o equilíbrio em diversas circunstâncias ${ }^{6-8}$. Essas estratégias de movimento são impostas tanto no plano sagital (ântero-posterior), como no plano frontal (médio lateral). As estratégias utilizadas no plano sagital são: estratégias de tornozelo, quadril e passo e estas são ativadas pelo recrutamento de determinados músculos, ocorrendo a sinergia destes de acordo com a tarefa a ser realizada. Já, no plano frontal as estratégias ocorrem principalmente no quadril, tronco e, no tornozelo ocorre apenas se a superfície é estreita ${ }^{9-11}$.

O treino de equilíbrio proporciona ao paciente com PC, o aumento do recrutamento muscular para a manutenção da postura em pé, promovendo, assim melhor ajuste postural ${ }^{10,12,13}$. A seleção dessas estratégias dependerá da superfície em que o paciente se encontra, da velocidade de desestabilização e também das aferências periféricas disponíveis. Portanto, o treino de equilíbrio em várias tarefas e situações permitirá a utilização das informações somatossensoriais, visuais e vestibulares, como também a utilização de diferentes estratégias motoras ${ }^{13}$.

Estudos realizados por diversos pesquisadores $^{7,14-17}$ observaram que crianças com PC diparética espástica apresentam déficits na adaptação sensorial e também apresentam uma maior dependência do retorno visual para a manutenção da postura correta. Por isso, a importância de se trabalhar o equilíbrio em diferentes ambientes sensoriais.

Desafios adicionais que encorajam o uso da informação vestibular remanescente podem ser introduzidos pela disponibilização gradual da informação visual mais acurada, como uma referência de orientação. Isto pode ser adquirido pela utilização de superfícies diferentes. Primeiro uma superfície firme que encoraje o paciente a utilizar mais as informações somatossensoriais. Depois uma superfície de espuma, que é menos acurada e a criança utiliza a informação visual e vestibular. Já, se a informação visual for retirada o paciente terá que utilizar a informação vestibular para o restabelecimento do equilíbrio ${ }^{16-18}$.

Os efeitos da experiência em relação ao treino de equilíbrio com respostas automáticas da postura observou-se que o treinamento repetitivo do equilíbrio pode modificar os ajustes posturais devido a maturação e experiência motora, sugerindo que os ajustes posturais podem ser aprendidos ${ }^{19}$.

Por isso, os treinamentos de equilíbrio que exigem repetição e modificação do ambiente são importantes para a prática e promovem melhora no desempenho do controle postural de crianças com $\mathrm{PC}^{13,20}$.

A prática e a experiência levam a mudanças relativamente permanentes na capacidade de produzir uma ação hábil. Além disso, a aprendizagem motora envolve a aquisição de novas estratégias para movimentar como também a busca de solução para uma tarefa que surge da interação entre o indivíduo, a tarefa e o ambiente ${ }^{13,21,22}$.

Essa aprendizagem motora e a habilidade em recuperar a estabilidade após perturbações podem também causar implicações clínicas importantes em crianças com idade de 7-12 anos de idade. Pois sabe-se que as crianças com desenvolvimento normal devem ter atingido os padrões de um adulto no equilíbrio e na estabilidade por volta dos 7 aos 10 anos de idade e, as crianças com PG são atrasadas neste aspecto $^{21}$.

Em estudos realizados com crianças com PC, mesmo com idade acima de 7 anos, observa-se que são hábeis para fazerem modificações nos ajustes posturais ${ }^{21}$. Por isso, a idade das crianças foi um critério importante, assim como também a escassez de estudos de treino de equilíbrio em crianças com PC. 
O objetivo deste estudo foi verificar os efeito do treino de equilíbrio como conduta fisioterapêutica, no ajuste postural e nas atividades funcionais em ortostatismo de crianças com PG diparética espástica.

\section{MÉTODO}

Neste estudo de casos participaram 4 pacientes, sendo considerados critérios de inclusão: diagnóstico clínico de PG diparética espástica, em intervenção fisioterapêutica no Centro de Reabilitação Lar Escola São Francisco (LESF); idade entre 7-12 anos; espasticidade leve a moderada (grau 1-2) em MMII, de acordo com a Escala Modificada de Ashworth; realizar marcha independente com ou sem um dispositivo de auxílio; ortostatismo independente por pelo menos 30 segundos; concordância e assinatura do Termo de Consentimento Livre e Esclarecido dos pais ou responsáveis das crianças envolvidas na pesquisa. Os critérios de exclusão foram: presença de deformidades osteo-articulares estruturadas em quadril, joelho e tornozelos; realização de cirurgia ou bloqueio químico neuromuscular há menos de 6 meses; pacientes com alterações nas funções cognitivas que impedissem a colaboração e compreensão nas atividades propostas.

No período pré e pós intervenção, foram realizadas as avaliações iniciais e finais respectivamente, sendo compostas pela avaliação do equilíbrio funcional com o teste de Equilíbrio de Berg, e pela avaliação da função motora através do Gross Motor Function Measure (GMFM), contendo as dimensões $\mathrm{D}$ (em pé) e $\mathrm{E}$ (andar, correr, pular).

O teste de equilíbrio de Berg avalia o desempenho do equilíbrio funcional em 14 itens comuns à vida diária. A pontuação máxima é de 56 e cada item possui uma escala ordinal de cinco alternativas que variam de 0 a 4 pontos. Os pontos são baseados no tempo em que uma posição pode ser mantida, na distância em que o membro superior é capaz de alcançar à frente do corpo e no tempo para completar uma tarefa. Este teste leva aproximadamente 15 minutos para ser executado ${ }^{23-26}$.A escala atende a várias propostas: descrição quantitativa da habilidade, acompanhamento do progresso dos pacientes e avaliação da efetividade das intervenções na prática clínica e em pesquisas ${ }^{23}$.

Neste trabalho foram avaliados os 14 itens do teste de equilíbrio de Berg.

Após a avaliação da escala de equilíbrio de Berg avaliou-se a função motora grossa através do
GMFM que é um instrumento de observação padronizado, criado e aprovado para quantificar e mensurar a função motora grossa que ocorre com o passar do tempo nas crianças com paralisia cerebral.

O teste de GMFM inclui 88 itens que avaliam a função motora em cinco dimensões: (A) deitar e rolar; (B) sentar; (C) engatinhar e ajoelhar; (D) ficar em pé; e (E) andar, correr e pular ${ }^{27,28}$.

Os itens são agrupados de acordo com a seqüência do desenvolvimento motor normal. Para fins de pontuação, os itens são agregados para representar cinco áreas separadas da função motora.

Neste estudo foram aplicadas as dimensões D e E do GMFM que compreendem as atividade de ficar em pé, de andar, correr e pular. O item D é composto de 13 itens numerados de 52 a 64 . O item E é composto por 24 itens numerados de 65 a 88 . Cada item pode ser pontuado de 0 a 3 , que correspondem respectivamente a: 0 não consegue realizar a atividade; 1 inicia a atividade $(<10 \%$ da tarefa); 2 completa parcialmente $(10 \mathrm{a}<100 \%)$ e 3 completa a tarefa $(100 \%)$.

Para a pontuação dos domínios D e E foi realizada a soma dos itens obtendo assim a pontuação total de cada domínio. Desse modo, uma pontuação percentual é calculada para cada dimensão (pontuação da criança/ pontuação máxima X 100\%). Uma pontuação total, ou seja, o escore total é obtida pela adição da pontuação percentual para cada dimensão dividida por 2, devido ao uso dos itens D e E. Nesta escala, qualquer mudança na pontuação total será considerado melhora ou não melhora, dependendo assim da variação da pontuação na avaliação inicial e final ${ }^{27,28}$.

O tratamento fisioterapêutico foi baseado em uma proposta de intervenção com 7 atividades que visavam o treino de equilíbrio em postura ortostática com diferentes bases de apoio e utilização de estratégias sensoriais e motoras. Foram realizadas doze sessões de treino de equilíbrio por um período de três meses consecutivos, com uma freqüência de uma vez por semana e duração de uma hora cada.

\section{Intervenção}

1- Paciente em pé com apoio bipodal em uma superfície estável e o terapeuta realiza a desestabilização unidirecional e bidirecional com intensidade leve no início e depois evoluindo com uma intensidade maior. Primeiramente realiza-se com os olhos abertos e em seguida com os olhos fechados. 
2- Paciente em pé em uma superfície estável com uma base estreita e o terapeuta realiza a desestabilização unidirecional e bidirecional com intensidade leve no início e depois evoluindo com uma intensidade maior. Primeiramente realiza-se com os olhos abertos e em seguida com os olhos fechados.

3- Paciente em pé em uma superfície estável são realizadas tarefas funcionais com MMSS de acordo com a capacidade funcional de cada criança. Por exemplo, jogar bola.

4- Paciente em pé sobre uma superficie de espuma de densidade média, com os pés paralelos e ligeiramente afastados. O terapeuta realiza movimentos no sentido ântero-posterior e latero-lateral, com os olhos abertos e fechados para promover o ajuste postural. A desestabilização é iniciada com leve intensidade e aumentada progressivamente.

5- Paciente em pé no mini-tramp são realizadas atividades com MMSS, como, por exemplo, jogar bola, exigindo o ajuste postural da criança.

6- Paciente em pé na plataforma de equilíbrio, com os pés paralelos e ligeiramente afastados, com um espelho à frente. $\mathrm{O}$ terapeuta realiza movimentos no sentido ântero-posterior para promover o ajuste postural. A desestabilização é iniciada com leve intensidade e aumentada progressivamente.

7- Paciente em pé na plataforma de equilíbrio, com os pés paralelos e ligeiramente afastados, com um espelho à frente. O terapeuta realiza movimentos no sentido látero-lateral para promover o ajuste postural. A desestabilização é iniciada com leve intensidade e aumentada progressivamente.

\section{RESULTADOS}

De acordo com a pontuação no teste de equilíbrio de Berg, verificou-se a melhora de todos os pacientes, sendo a variação da pontuação de 4 a 7 pontos (Tabela 1).

Na mensuração do GMFM, todos os pacientes apresentaram melhora, sendo a variação de porcentagem de $3.5 \%$ a $10 \%$ (Tabela 2 ).

Tabela 1. Variação da pontuação inicial e final no Teste de Equilíbrio de Berg.

\begin{tabular}{|l|c|c|c|}
\hline Pacientes & $\begin{array}{c}\text { Avaliação } \\
\text { Inicial }\end{array}$ & $\begin{array}{c}\text { Avaliação } \\
\text { Final }\end{array}$ & $\begin{array}{c}\text { Variação da pontuação } \\
\text { da escala de Berg entre a } \\
\text { avaliação inicial e final }\end{array}$ \\
\hline Paciente 1 & 46 pontos & 53 pontos & 7 pontos \\
\hline Paciente 2 & 44 pontos & 51 pontos & 7 pontos \\
\hline Paciente 3 & 44 pontos & 49 pontos & 5 pontos \\
\hline Paciente 4 & 49 pontos & 53 pontos & 4 pontos \\
\hline
\end{tabular}

Tabela 2. Variação da porcentagem do escore meta da mensuração da avaliação inicial e final do GMFM.

\begin{tabular}{|l|c|c|c|}
\hline Pacientes & Avaliação Inicial & Avaliação Final & $\begin{array}{c}\text { Variação da } \\
\text { porcentagem }\end{array}$ \\
\hline Paciente 1 & $86,5 \%$ & $92 \%$ & $5,5 \%$ \\
\hline Paciente 2 & $73,5 \%$ & $79,5 \%$ & $6 \%$ \\
\hline Paciente 3 & $73 \%$ & $83 \%$ & $10 \%$ \\
\hline Paciente 4 & $80,5 \%$ & $84 \%$ & $3,5 \%$ \\
\hline
\end{tabular}

\section{DISCUSSÃO}

Os resultados obtidos neste estudo são compatíveis com os dados de trabalhos semelhantes a este realizados em crianças com PC diparética espásti$\mathrm{ca}^{18,21}$.

Na variação da pontuação do Teste de Equilíbrio de Berg, observou-se uma variação mínima de 4 pontos e a máxima de 7 pontos. E na pontuação do GMFM foi observada uma mudança que variou de $3.5 \%$ a $10 \%$ da avaliação inicial e final. Nestas duas escalas foram considerados que qualquer variação na pontuação da avaliação inicial e final é indicativo de melhora. Sendo assim, sugere-se a melhora do equilíbrio e atividades funcionais em ortostatismo de crianças com PC diparética espástica participantes deste estudo.

Como a proposta do estudo foi mensurar o ajuste postural em ortostatismo, foi realizada apenas as avaliações das dimensões D e E do GMFM, encontradas também em outros estudos ${ }^{21}$.

Neste estudo, observou-se que o treino de equilíbrio promoveu a melhora do ajuste postural em ortostatismo, como também a melhora funcional do equilíbrio. Assim, pode-se sugerir que é possível observar modificações nas crianças com faixa etária mais avançada. Portanto, a continuidade da prática é relevante na recuperação do equilíbrio e da estabilidade.

Com base nos dados já observados, alguns autores sugerem implicações clínicas importantes na habilidade em recuperar a estabilidade após perturbações, sendo modificáveis em crianças com faixa etária entre 7-12 anos ${ }^{21,29}$.

Estudo realizado por Shumway-Cook et $\mathrm{al}^{21}$ obteve melhora na pontuação do GMFM, através do treino intenso na plataforma de força. Os achados destes estudos concordam com estudos de Russel et $\mathrm{al}^{28}$, Trahan et al ${ }^{30}$ que também verificaram uma melhora na porcentagem da pontuação do GMFM, em crianças com PG. 
Baseado nos resultados pode-se sugerir que a especificidade do treinamento deste estudo, através da vivência do equilíbrio estático e dinâmico em diversas superfícies, com diferentes estratégias sensoriais, ambientais, promove assim uma melhora do ajuste postural.

Na abordagem sistemática, a análise do desenvolvimento motor considera a contribuição de todos os elementos dentro do sistema nervoso em harmonia com a contribuição de outros subsistemas, como o sistema muscular e esquelético ${ }^{19,31}$. Esta abordagem também considera a informação do meio ambiente como sendo crítica na contribuição para o controle postural da criança.

Portanto, o tratamento baseado na promoção da resposta muscular, antecipação, adaptação motora e sensorial, a variabilidade do ambiente como também o aprendizado motor proporcionam a melhora do ajuste postural e um melhor equilíbrio durante atividades funcionais.

Achados bibliográficos observados nos estudos práticos de Carr et al ${ }^{13}$ revelam que a flexibilidade de condições ambientais e temporais do ambiente faz com que ocorra, também a flexibilidade no desempenho motor e postural. Os exercícios que promovem deslocamentos de massa corporal, maior velocidade e maior complexidade (diferentes atividades) aumentarão a confiança, a eficiência e segurança durante atividades que exijam um controle postural eficiente ${ }^{7,15,32,33}$. Portanto, neste estudo, os fatores ambientais que correspondem as diferentes superficies e estimulação sensorial multimodal podem ter influenciado na melhora do ajuste postural.

Os dados observados nos estudos de Ayres ${ }^{17}$, Valade et $\mathrm{al}^{12}$, Horak et $\mathrm{al}^{18}$ revelam que o treino de equilíbrio fornece e controla a entrada de estímulos sensoriais, de tal forma que a criança espontaneamente forma as respostas adaptativas que integram todas as sensações. Estes estímulos sensoriais em todas as articulações, pele e músculos da criança, irão gerar respostas motoras e sensoriais para o restabelecimento do equilíbrio. Conforme as crianças gradualmente dominam estas sensações e respostas, o seu cérebro aprende a modular a atividade sensorial e forma uma percepção corporal mais precisa.

Portanto, o tratamento de equilíbrio utilizando diferentes superfícies e materiais que estimulam as informações sensoriais irá promover melhora da resposta do ajuste postural em pé.

A prática é essencial para o aprendizado efetivo de tarefas complexas e também para a melhora da função motora ${ }^{19,22}$. Esses dados concordam com os resultados obtidos neste estudo, pois observou-se na avaliação do GMFM a melhora da função motora grossa através do treinamento de equilíbrio realizado durante três meses.

As crianças com PG diparética espástica necessitam da oportunidade de praticar o ajuste postural sob uma variedade de situações relevantes, fazendo com que ocorra o desenvolvimento da habilidade $\mathrm{e}^{11,13}$.

Portanto, é importante que na intervenção fisioterapêutica baseada na melhora do equilíbrio em ortostatismo de crianças com PC diparética espástica seja enfatizada a estimulação sensorial multimodal, pelo treino em diferentes superfícies e estratégias sensoriais e pela variabilidade da prática.

\section{CONCLUSÃO}

A fisioterapia com ênfase no treino de equilíbrio melhorou o ajuste postural em ortostatismo e as atividades funcionais de pacientes com paralisia cerebral diparética espástica deste estudo.

Porém, algumas limitações foram encontradas no decorrer do estudo. Algumas em decorrência da falta de métodos fidedignos, devido ao alto custo, para a mensuração do equilíbrio, como por exemplo a plataforma de força e a eletromiografia, utilizados em alguns estudos para avaliar a resposta motora e sensorial de crianças com PC para o restabelecimento do equilíbrio.

Finalmente, devido ao número pequeno de 4 pacientes neste estudo, coloca-se a necessidade de aumentar a amostra de sujeitos para averiguar valores mais significativos através de uma análise estatística.

\section{REFERÊNCIAS BIBLIOGRÁFICAS}

1. Piovesana AMSG. Paralisia Cerebral: contribuição do estudo por imagem. In: Souza AMC, Ferrareto I (orgs). Paralisia Cerebral: aspectos práticos. São Paulo: Memnon, 1998, p8-28.

2. Rosen MG, Dickinson JC. The incidence of Cerebral Palsy. Am J Obstetr Gynecol 2000;167:417-423.

3. Rotta NT. Paralisia Cerebral, novas perspectivas terapêuticas. J Ped 2002;78(suppl.1):48-54.

4. Katsetos CD, Legido A. Paralises Cerebral nuevos conceptos etiopatogenicos. Rev Neurol 2003;36:2,157-165.

5. Leite JMRS, Prado GF. Paralisia Cerebral - aspectos fisioterapêuticos e clínicos. Rev Neurocienc 2004;12(1):41-45.

6. Shumway-Cook A, Woollacott MH. Controle Motor: teoria e aplicações práticas. São Paulo: Manole, 2003, 592p.

7. Cherng R, Su FC, Chen IIJ, Kuan TS. Performance of static standing balance in children with spastic diplegic cerebral palsy under altered sensory environments. Am J Phys Med Rehabil 1999; 78(4):336-343.

8. Lundy-Ekman L. Neurociência: fundamentos para reabilitação. Rio de Janeiro: Guanabara Koogan, 2000, p153-156. 
9. Woollacott MH, Burtner P. Neural and musculoskeletal contribuitions to the development of stance balance control in typical children and in children with cerebral palsy. Ac Ped 1996; 416:58-62.

10. Rothwel J. Controle motor normal. In: Stokes M. Neurologia para Fisioterapeutas. São Paulo: Editorial Premier, 2000, p25-38.

11. Mochizuki L, Amadio AC. As funções do controle postural durante a postura ereta. Rev. Fisioter 2003; 1:7-15.

12. Valade D, Bleton JP, Chevalier AM. Rééducation de la posture et de l'equilibre. Encycl Méd Chir Kinésither 1998;26-452 a, pl4.

13. Carr J, Shepherd RB. Um modelo de aprendizagem motora para a reabilitação In: Shepherd RB, Carr J. Ciência do Movimento: fundamentos para a fisioterapia na reabilitação. São Paulo: Manole, 2003, p33-110.

14. Nashner LM, Shumway-Cook A, Marin O. Stance posture control in select groups of children with cerebral palsy: deficits in sensory organization and muscular coordination. Exp Brain Res 1983; 49:393-409.

15. Rose J, Wolff DR, Jones VK, Bloch DA, Oehlert JW, Gamble JG. Postural balance in children with cerebral palsy. Dev Med Child Neurol 2002; 44:58-63.

16. Shumway-Cook A, Horak F, Black OF. A critical examination of vestibular function in motor-impaired learning-disable children. Inter J Ped Otorhinol 1987; 14:21-30.

17. Ayres AJ. Sensory integration and the child. Los Angeles: Western Psycological Services, 1979, 191p.

18. Horak FB, Henry SM, Shumway-Cook A. Postural pertubations: new insights for treatment of balance disorders. Phys Ther 1997; 77(5):17-32

19. Sveistrup H, Woollacott MH. Pratice modifies the developing automatic postural response. Exp Brain Res 1997; 114:33-43.

20. Shepherd RB. Fisioterapia em Pediatria. 3.ed. São Paulo:Santos, 1995, 420p.

21. Shumway-Cook A, Hutchinson S, Kartin D, Price R, Woollacott M. Effect of balance training on recovery of stability in children with cerebral palsy. Dev Med Child Neurol 2003;.45:591-602.

22. Walker C, Bhrouwer BJ, Culham EG. Use of visual feedback in retraining balance folowing acute stroke. Phys Ther 2000; 80(9):886-895.
23. Berg K, Wood-Dauphinée S, Willians J, Gayton D. Measuring balance in the elderly: preliminary development of na instrument. Physiother Can 1989; 41:304-311.

24. Kembhavi G, Darrah J, Magill-Evans J, Loomis J. Using the berg balance scale to distinguish balance abilities in children with cerebral palsy. Ped Phys Ther 2002; 14(2):92-99.

25. Franjoine MR, Gunther JS, Taylor, MJ. Pediatric Balance Scale: A modified version of the Berg Balance Scale for the Schooll-age Child with mild to moderate motor impairment. Ped Phys Ther 2003;15(2):114128.

26. Miyamoto ST, Lombard Junior I, Berg KO, Ramos LR, Natour J. Brazilian version of the Berg balance scale. Braz J Med Biol Res 2004; 37(9):1411-1421.

27. Russell DJ, Avery LM, Rosenbaum PL, Raina PS, Walter D, Palisano RJ. Improved Scaling of the Gross Motor Function Measure for Children With Cerebral Palsy; evidence of reliability and validity. Phys Ther 2000; 80(9):873-885.

28. Russell DJ, Rosenbaum PL, Gowland C. Manual for the gross motor function meassure. 2nd Ed. Ontario: Mc Master University, 1993, 244p.

29. Shumway-Cook A, Woollacott MH. The growth of estability postural control from developmental perpectivel. J Mot Behav 1985; 17:131147.

30. Trahan J, Malouin F. Changes in the gross motor function measure in children with cerebral palsy: an eight month follow up study. Ped Phys Ther 1999; 11:12-17.

31. Woollacott MH, Burtner J, Jensen J, Jasiewicz N, Roncesvalles MN, Sveistrup H. Development of postural response during standing in healthy children with spastic diplegia. Neurosci Biobeh Rev 1998; 4:583589 .

32. Horak FB, Shupert CL, Dietz V, Horstmann G. Vestibular and somatosensory contributions to responses to head and body displacements in stance. Exp Brain Res 1994; 100:93-106.

33. Riemann BL, Lephart SM. The Sensorimotor System, Part II: the role of proprioception in motor control and functional joint stability. $\mathrm{J}$ Athl Train 2002; 1:.80-84. 